\title{
Diversity and biotechnological potential of culturable bacteria from Brazilian mangrove sediment
}

\author{
Armando C. F. Dias - Fernando Dini Andreote $\cdot$ Francisco Dini-Andreote \\ Paulo T. Lacava · André L. B. Sá · Itamar S. Melo · João L. Azevedo · \\ Welington L. Araújo
}

Received: 22 December 2008/ Accepted: 28 February 2009/Published online: 14 March 2009

(C) Springer Science+Business Media B.V. 2009

\begin{abstract}
Mangrove ecosystems are environments subject to substantial degradation by anthropogenic activities. Its location, in coastal area, interfacing the continents and the oceans makes it substantially important in the prospection for biotechnological applications. In this study, we assessed the diversity of culturable bacteria present over the seasons at two depths $(0-10$ and $30-40 \mathrm{~cm})$ in a mangrove sediment and in a transect area from the land to the sea. In total, 238 bacteria were isolated, characterized by Amplified Ribosomal DNA Restriction Analysis (ARDRA) and further identified, by Fatty Acid Methyl Esther (FAME-MIDI), into the orders of Vibrionales, Actinomycetales and Bacillales. Also the ability of the isolates in producing economically important enzymes (amylases,
\end{abstract}

A. C. F. Dias · A. L. B. Sá

Center of Biotechnological Researches, ICB-IV, University of São Paulo, Av. Prof. Lineu Prestes, 1374, São Paulo, SP 05508-900, Brazil

A. C. F. Dias

e-mail: dias147@gmail.com

A. C. F. Dias - F. D. Andreote - F. Dini-Andreote

P. T. Lacava · A. L. B. Sá · J. L. Azevedo · W. L. Araújo Department of Genetics, Escola Superior de Agricultura "Luiz de Queiroz", University of São Paulo, ESALQ/USP, Av. Pádua Dias, 11, Piracicaba, SP 13400-970, Brazil

F. D. Andreote $(\varangle)$. I. S. Melo

Laboratory of Environmental Microbiology, CNPMA, Embrapa Meio Ambiente Rodovia SP 340, Km 127,5, Jaguariúna, SP 13820-000, Brazil

e-mail: fdandreo@gmail.com

W. L. Araújo

Center of Biotechnological Researches, Universidade de Mogi das Cruzes, Av. Dr. Cândido Xavier de Almeida Souza, 200,

Mogi das Cruzes, SP 08780-911, Brazil proteases, esterases and lipases) was evaluated and the order Vibrionales was the main enzymatic source.

Keywords Environment - Enzymes · ARDRA · FAME-MIDI

\section{Introduction}

The mangrove is a tropical coastal biome, located in the transition zone between land and sea, where the vegetation is dominated by a particular group of plant species (Zhou et al. 2006). This ecosystem is characterized by periodic tidal flooding which makes environmental factors such as salinity and nutrient availability highly variable, resulting in unique and specific characteristics (Holguin et al. 2006).

A phylogenetic and functional description of microbial diversity in the mangrove ecosystem has not been addressed to the same extent as that of other environments (Zhou et al. 2006). A more thorough description of the bacterial diversity and distribution in a mangrove would improve our understanding of bacterial functionality and microbial interactions found in that ecosystem (Kathiresan and Selvam 2006). Given the particular conditions of a mangrove and the adaptation of bacterial species to such conditions, the microbiota represents an important potential source of biotechnological resources to be exploited (Sivaramakrishnan et al. 2006). This biotechnological potential includes characterization of novel enzymes from previously uncharacterized bacterial species, with useful applications to many aspects of human life, from agriculture to medicine (Lageiro et al. 2007). The present work aimed to elucidate the bacterial diversity and the potential usefulness of microbiota inhabiting sediments of a well preserved mangrove in Ilha do Cardoso (Cananéia, Brazil). 
Following the determination of the main culturable bacterial groups, an enzyme production analysis was conducted to address this diversity as a potential source for biotechnology.

\section{Materials and methods}

Mangrove and sampling points

The study was developed in a transect area, $340 \mathrm{~m}$ in length, along the flood gradient of the mangrove from Ilha do Cardoso (Cananéia-SP, Brazil). Five samples were collected from each of five different points (P1-P5) distributed over a transect. P1 had the coordinates $25^{\circ} 05^{\prime} 1$, $87^{\prime \prime} \mathrm{S} 47^{\circ} 57^{\prime} 41,70^{\prime \prime} \mathrm{W}$ and $\mathrm{P} 5$ was located at $25^{\circ} 05^{\prime} 12,61^{\prime \prime} \mathrm{S}$ $47^{\circ} 57^{\prime} 41,21^{\prime \prime} \mathrm{W}$. The remaining points, $\mathrm{P} 2, \mathrm{P} 3$ and $\mathrm{P} 4$, were equidistantly distributed over the transect and separated from one another by $70 \mathrm{~m}$. Samples were collected in stainless steel sampler cylinder $(100 \mathrm{~cm}$ long and $7 \mathrm{~cm}$ in diameter), transferred to labeled plastic bags and stored in boxes. They were collected from depths of 0 to 10 and 30 to $40 \mathrm{~cm}$ during two different seasons; winter (12th July 2005) and summer (19th March 2006).

\section{Bacterial isolation from mangrove sediment}

Five sub-samples of sediment were collected from each point and were mixed into a composite sample. Aliquots of $10 \mathrm{~g}$ from each sediment mixture were added into $90 \mathrm{ml}$ sterile water and shaken for $1 \mathrm{~h}$ at $150 \mathrm{rev} / \mathrm{min}$. From the resulting suspensions, tenfold serial dilutions were prepared in sterilized water and $100 \mu \mathrm{l}$ aliquots from dilutions $10^{-3}, 10^{-4}$ and $10^{-5}$ were plated onto a non-selective culture media containing Tryptone Soy Broth (TSB; Dif$\mathrm{co}^{\mathrm{Tm}}$, Sparks, MD, USA) at $5 \%$ of the recommended amount $\left(1.5 \mathrm{~g} \mathrm{l}^{-1}\right)$, amended with $1.8 \% \mathrm{NaCl}$ and supplemented with $50 \mathrm{mg}$ Benomyl $\mathrm{ml}^{-1}$. The plates were incubated at $28^{\circ} \mathrm{C}$ and the development of bacterial colonies was monitored for a 14-day period, with colonyforming unit (c.f.u.) estimation conducted per gram of mangrove sediment. The results were evaluated by ANOVA and average comparisons using Tukey's test at a 95\% confidence level $(P<0.05)$.

\section{ARDRA characterization of bacterial isolates}

The genotypic characterization of the bacterial isolates, into ribotype groups, was based on the ARDRA patterns. Bacterial DNA was extracted from each isolate using the Wizard DNA extraction kit (Promega, Madison, USA), according to the manufacturer's instructions. The $16 \mathrm{~S}$
rDNA gene was amplified from DNA extracted from each strain using the primers 27F and 1378R (Heuer et al. 1997). The PCR products were subjected to restriction analysis with $1 \mathrm{U}$ of a $4 \mathrm{bp}$ recognition site endonuclease HaeIII and further separated by electrophoresis on a $2.4 \%$ agarose gel (stained with ethidium bromide and visualized by UV light). Distinct cleavage patterns were considered as different ribotypes identified by FAME.

\section{Bacterial identification by MIDI-FAME}

Isolates from each ribotype (10\% of the total) were selected and each strain was inoculated on tryptone soy broth agar (TSBA; Difco $^{\mathrm{Tm}}$, Sparks, MD, USA) for identification by MIDI-FAME. The FAME analysis was conducted by gas chromatography with automatic injector and Flame Ionization Detector (FID) (Agilent, 6850 and 7683). The output profile was organized into a chromatogram and a report generated by the Microbial Identification System software (Sherlock TSBA40 library; MIDI Inc., Newark, DE, USA). The final results, based on similarities found between the database and the nominated areas, identified the isolates.

Enzymatic production of isolates

The phenotypic characterization of isolates was conducted to evaluate the biotechnological potential of culturable bacteria found in mangroves. It was determined by a batch of assays, evaluating the production of amylases, proteases, esterases and lipases. In each of these analyses, the enzyme activities were measured by the value obtained subtracting the halo size (enzyme activity) by the diameter of the bacterial colony, generating a semi-quantitative approach.

\section{Determination of amylolytic activity}

Isolates were grown on 5\% TSBA medium containing $1 \%$ starch, and incubated at $28^{\circ} \mathrm{C}$ for $72 \mathrm{~h}$. After bacterial growth, $5 \mathrm{ml}$ of $1 \%$ iodine solution was added to each plate and the presence of a stainless zone around the colony indicated amylase production (Hankin and Anagnostakis 1975).

\section{Determination of proteolytic activity}

Bacterial isolates were inoculated onto plates containing agar medium and incubated at $28^{\circ} \mathrm{C}$ for 5 days. After colony development, the medium was covered with Frasier solution for $2 \mathrm{~min}$. Proteolytic activity was detected by the visualization of a transparent area around the colonies (Smibert and Krieg 1994). 
Determination of esterase activity

Esterase activity was detected using a culture medium described by Sierra (1957). After sterilization of the culture medium, Tween 80 (previously sterilized) was added to a final aqueous concentration of $1 \%(\mathrm{v} / \mathrm{v})$. After inoculation, bacterial growth was observed and the presence of degradation zone was considered indicative of esterase activity.

\section{Determination of lipolytic activity}

In order to detect lipolytic activity, a similar methodology was used as described above for esterase activity, with the exception that Tween 80 was replaced by Tween 20 . The presence of a degradation zone was noted after bacterial growth (Sierra 1957).

\section{Results and discussion}

Isolation and characterization of the culturable bacterial community

Bacterial isolations were performed during two seasons and at different sediment depths, allowing a comprehensive description of the culturable microbiota found in this ecosystem. The results revealed a diverse and dense bacterial community inhabiting the mangrove sediment. The amendment of the medium with $1.8 \%$ of $\mathrm{NaCl}$ ensured the selection of bacteria typically found in mangrove ecosystems. The bacterial diversity found in the isolations from the two seasons revealed to be statistically different $(P<0.05)$. In the winter, the number of bacteria had a $\log$ value of 6.85 c.f.u. $\mathrm{g}^{-1}$ of sediment, while in the summer the value was $6.22 \mathrm{log}$ c.f.u. $\mathrm{g}^{-1}$ of sediment. However, no significant differences were observed between samples collected from different depths. Almeida et al. (2007) found similar results when studying four estuaries during different seasons.

\section{ARDRA typing and identification of isolates}

A total of 238 colonies (159 from the winter and 79 from the summer) were chosen for further characterization with ARDRA analysis, generating ten distinct cleavage patterns (named ribotypes). Samples containing around 10\% of isolates from each ribotype were used for bacterial identification by the FAME-MIDI. FAME identification is highly reliable for similarities higher than 0.70 at species level, while lower levels can affiliate isolates to higher taxonomic groups, like genus or families (Heyrman et al. 1999). Also, in some phylogenetic groups, this technique is less sensitive, like for Vibrio, where all isolates were classified only as Vibrio sp. Besides it, the identified groups were classified into three orders; Actinomycetales (ribotypes B, G, $\mathrm{H}, \mathrm{I}$ and J), Bacillales (ribotypes C, D, E and F) and Vibrionales (ribotype A) (Fig. 1). The order Actinomycetales was the most diverse group, composed of seven different genera; Brevibacterium, Dermabacter, Kocuria, Kytococcus, Microbacterium, Nesterenkonia and Rothia. The order Bacillales was represented by four genera: Bacillus, Kurthia, Paenibacillus and Staphylococcus; while the order Vibrionales was represented by the genera Vibrio and Listonella (Table 1).

By analysing the ribotype frequencies and associating them with the identities determined by FAME-MIDI, it was possible to make predictions about the population fluctuation of each order over the year and at different depths (Fig. 1). A higher frequency of isolates from the order Vibrionales was clearly visible in winter samples, while isolates from orders Bacillales appeared to be more abundant in summer and at depth of $0-10 \mathrm{~cm}$. The order Actinomycetales was more constant through the considered changes (Fig. 1).

The bacterial groups that were detected in the mangrove sediment are commonly found in marine and estuarine environments (Holguin and Bashan 1996; Thompson et al. 2004; Sousa et al. 2006; Stevens et al. 2007). The order Vibrionales was previously described from marine environments responding to variations in weather or to environmental factors such as salinity (Thompson et al. 2004; Sousa et al. 2006). The order Actinomycetales is widely found in soils, where it shows little response to abiotic factors (Tiago et al. 2004). Considering the species occurrence within the Actinomycetales order, although the genera Nesterenkonia, Kytococcus, Kocuria and Rothia were isolated at low densities, these groups were expected to be residents of mangrove sediments, according to previously described results (Tiago et al. 2004). Concerning the order Bacillales, representatives of this group were

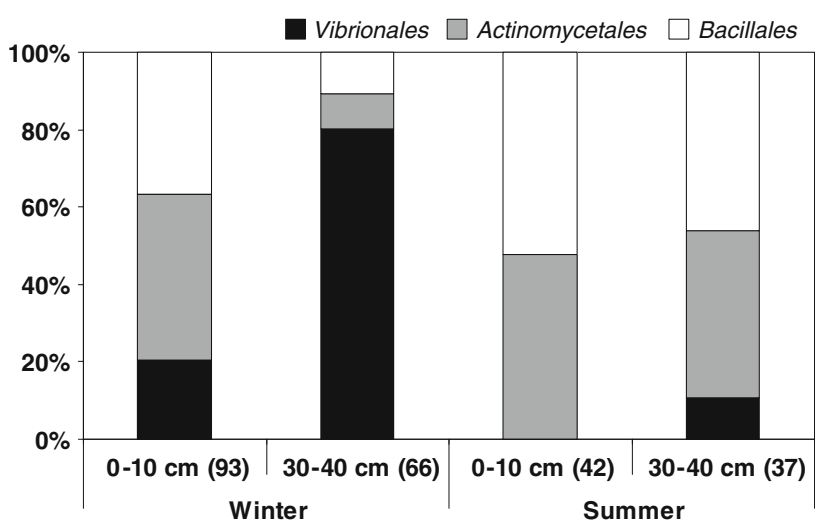

Fig. 1 Relative abundance of the three orders represented by the ten ribotypes found among the bacterial isolates from the mangrove sediments sampled in different seasons and at two depths. The number after the depth, in parentheses, represents the number of analyzed isolates 
Table 1 Identification by FAME-MIDI of ribotypes constituted by isolates from mangrove sediments

\begin{tabular}{|c|c|c|c|c|}
\hline Isolate & Ribotype & Order & Best match & Similarity (\%) \\
\hline $3 \mathrm{~B} 31$ & A & Vibrionales & Listonella sp. & 0.852 \\
\hline $5 B 324$ & A & Vibrionales & Listonella sp. & 0.739 \\
\hline $5 \mathrm{~B} 338$ & A & Vibrionales & Vibrio aestuarianus & 0.852 \\
\hline 1B318 & A & Vibrionales & Vibrio fluvialis & 0.884 \\
\hline $1 \mathrm{~A} 312$ & A & Vibrionales & Vibrio sp. & 0.914 \\
\hline 1B321 & A & Vibrionales & Vibrio sp. & 0.866 \\
\hline 1B35 & A & Vibrionales & Vibrio sp. & 0.853 \\
\hline 3B34 & A & Vibrionales & Vibrio sp. & 0.867 \\
\hline $5 \mathrm{~A} 36$ & A & Vibrionales & Vibrio sp. & 0.929 \\
\hline $5 \mathrm{~B} 321$ & A & Vibrionales & Vibrio sp. & 0.807 \\
\hline $5 \mathrm{~B} 330$ & $\mathrm{~A}$ & Vibrionales & Vibrio sp. & 0.920 \\
\hline $5 \mathrm{~B} 337$ & A & Vibrionales & Vibrio sp. & 0.848 \\
\hline $5 \mathrm{~B} 350$ & A & Vibrionales & Vibrio sp. & 0.778 \\
\hline $1 \mathrm{~B} 2 \mathrm{~S}$ & $\mathrm{~A}$ & Vibrionales & Vibrio sp. & 0.882 \\
\hline $1 \mathrm{~B} 3 \mathrm{~S}$ & A & Vibrionales & Vibrio sp. & 0.816 \\
\hline $2 \mathrm{~A} 33$ & A & Vibrionales & Vibrio sp. & 0.782 \\
\hline 3A372 & $\mathrm{B}$ & Actinomycetales & Brevibacterium mcbrellneri & 0.628 \\
\hline $4 \mathrm{~A} 11 \mathrm{~S}$ & $\mathrm{~B}$ & Actinomycetales & Dermabacter sp. & 0.497 \\
\hline $5 \mathrm{~A} 1 \mathrm{~S}$ & B & Actinomycetales & Dermabacter sp. & 0.446 \\
\hline $1 \mathrm{~A} 362$ & $\mathrm{~B}$ & Actinomycetales & Microbacterium sp. & 0.600 \\
\hline 3A357 & B & Actinomycetales & Microbacterium sp. & 0.533 \\
\hline 3B311 & B & Actinomycetales & Microbacterium testaceum & 0.705 \\
\hline 1A362 & B & Actinomycetales & Microbacterium imperiale & 0.793 \\
\hline $2 \mathrm{~A} 17 \mathrm{~S}$ & B & Actinomycetales & Microbacterium imperiale & 0.700 \\
\hline $3 \mathrm{~A} 35$ & B & Actinomycetales & Microbacterium imperiale & 0.740 \\
\hline 1A364 & $\mathrm{C}$ & Bacillales & Bacillus cereus & 0.635 \\
\hline $5 \mathrm{~A} 328$ & $\mathrm{C}$ & Bacillales & Bacillus cereus & 0.671 \\
\hline $2 \mathrm{~B} 35$ & $\mathrm{C}$ & Bacillales & Bacillus mycoides & 0.559 \\
\hline $5 \mathrm{~A} 312$ & $\mathrm{C}$ & Bacillales & Bacillus pumilus & 0.630 \\
\hline 1A339 & $\mathrm{C}$ & Bacillales & Bacillus sp. & 0.523 \\
\hline $4 \mathrm{~B} 2 \mathrm{~S}$ & $\mathrm{C}$ & Bacillales & Bacillus sp. & 0.325 \\
\hline 1B39 & $\mathrm{C}$ & Bacillales & Bacillus subtilis & 0.550 \\
\hline $1 \mathrm{~A} 33$ & $\mathrm{C}$ & Bacillales & Bacillus sp. & 0.570 \\
\hline 1B313 & $\mathrm{C}$ & Bacillales & Bacillus sp. & 0.690 \\
\hline 3B39 & $\mathrm{C}$ & Bacillales & Paenibacillus sp. & 0.480 \\
\hline $4 \mathrm{~A} 16 \mathrm{~S}$ & $\mathrm{D}$ & Bacillales & Staphylococcus sp. & 0.611 \\
\hline 4A1S & $\mathrm{D}$ & Bacillales & Staphylococcus sp. & 0.778 \\
\hline $1 \mathrm{~A} 321$ & $\mathrm{E}$ & Bacillales & Paenibacillus pabuli & 0.704 \\
\hline $1 \mathrm{~A} 310$ & $\mathrm{E}$ & Bacillales & Paenibacillus sp. & 0.533 \\
\hline $1 \mathrm{~A} 328$ & $\mathrm{E}$ & Bacillales & Paenibacillus sp. & 0.618 \\
\hline $1 \mathrm{~A} 365$ & $\mathrm{~F}$ & Bacillales & Kurthia gibsonni & 0.554 \\
\hline $5 \mathrm{~A} 310$ & $\mathrm{~F}$ & Bacillales & Kurthia sibirica & 0.429 \\
\hline $1 \mathrm{~A} 322$ & $\mathrm{G}$ & Actinomycetales & Nesterenkonia sp. & 0.590 \\
\hline $3 \mathrm{~A} 33$ & $\mathrm{G}$ & Actinomycetales & Nesterenkonia halobia & 0.721 \\
\hline $3 \mathrm{~A} 11 \mathrm{~S}$ & $\mathrm{H}$ & Actinomycetales & Kytococcus sedentarius & 0.676 \\
\hline $3 B 14 S$ & $\mathrm{H}$ & Actinomycetales & Kytococcus sedentarius & 0.660 \\
\hline $5 \mathrm{~A} 9 \mathrm{~S}$ & $\mathrm{H}$ & Actinomycetales & Kytococcus sedentarius & 0.555 \\
\hline
\end{tabular}


Table 1 continued

\begin{tabular}{lllll}
\hline Isolate & Ribotype & Order & Best match & Similarity $(\%)$ \\
\hline 3B8S & I & Actinomycetales & Kocuria sp. & 0.647 \\
3B35 & J & Actinomycetales & Rothia sp. & 0.605 \\
\hline
\end{tabular}

The isolates codes are formed by the point of sampling (1-5), followed by the layer sampled (A and B indicate $0-10$ and $30-40 \mathrm{~cm}$, respectively) and the number of the isolate. In addition, those isolates with the letter $\mathrm{S}$ in the end were obtained by sampling at summer

previously reported in estuarine and mangrove environments (Holguin and Bashan 1996).

It is remarkable that, although culture-independent approaches did not commonly corroborate with culturebased analysis, the identified groups are also described in other studies based on culture-independent analysis in similar environments. In Canada, the bacterial community associated with several fish, mollusks, sediments and marine waters were analysed, revealing the presence of several genera that were also isolated in the present work. Such genera included Microbacterium spp., Kocuria spp., Bacillus spp. and Vibrio spp., as the dominant organisms in this environment (Schulze et al. 2006).

Enzyme production by isolated bacteria

The detection of these groups in marine and estuarine environments can be further explored as a source for

Table 2 Enzyme production by the isolates found in the mangrove sediment and classified into the three bacterial orders

\begin{tabular}{|c|c|c|c|c|c|}
\hline \multirow[t]{2}{*}{ Order } & \multirow[t]{2}{*}{ Isolate } & \multicolumn{4}{|l|}{ Enzymatic activity } \\
\hline & & Amilase & Protease & Esterase & Lipase \\
\hline \multirow[t]{30}{*}{ Vibrionales } & $1 \mathrm{~A} 311$ & ND & $4.24 \pm 0.12 \mathrm{~b}$ & ND & ND \\
\hline & $1 \mathrm{~B} 32$ & $5.13 \pm 0.07 \mathrm{a}-\mathrm{e}$ & ND & $2.97 \pm 0.28$ ab & $3.27 \pm 0.08 \mathrm{bc}$ \\
\hline & 1B35 & $6.44 \pm 0.75 \mathrm{a}-\mathrm{c}$ & $2.31 \pm 0.22 \mathrm{~cd}$ & ND & ND \\
\hline & $2 \mathrm{~A} 33$ & ND & $8.96 \pm 0.77$ a & $2.28 \pm 0.14 \mathrm{c}-\mathrm{f}$ & $2.74 \pm 0.11 \mathrm{~b}-\mathrm{g}$ \\
\hline & $2 \mathrm{~B} 324$ & $1.78 \pm 0.06 \mathrm{fg}$ & 0.00 & $2.53 \pm 0.27 \mathrm{a}-\mathrm{d}$ & $4.80 \pm 0.11 \mathrm{a}$ \\
\hline & $2 \mathrm{~B} 326$ & $5.46 \pm 0.30 \mathrm{a}-\mathrm{d}$ & 0.00 & $1.57 \pm 0.26 \mathrm{i}-\mathrm{k}$ & $1.91 \pm 0.08 \mathrm{~h}-\mathrm{k}$ \\
\hline & $2 \mathrm{~B} 327$ & 0.00 & $4.36 \pm 0.22 \mathrm{~b}$ & $1.89 \pm 0.11 \mathrm{e}-\mathrm{j}$ & $2.31 \pm 0.09 \mathrm{e}-\mathrm{k}$ \\
\hline & 2B331 & $6.65 \pm 1.04 \mathrm{ab}$ & ND & $2.24 \pm 0.46 \mathrm{c}-\mathrm{g}$ & $2.51 \pm 0.14 \mathrm{c}-\mathrm{i}$ \\
\hline & 2B38 & ND & 0.00 & $2.40 \pm 0.10 \mathrm{~b}-\mathrm{e}$ & $2.00 \pm 0.23 \mathrm{~g}-\mathrm{k}$ \\
\hline & $3 \mathrm{~B} 33$ & 0.00 & $9.48 \pm 0.64$ a & $2.19 \pm 0.17 \mathrm{c}-\mathrm{h}$ & $2.33 \pm 0.16 \mathrm{e}-\mathrm{k}$ \\
\hline & $4 \mathrm{~A} 312$ & ND & $3.35 \pm 0.53 b c$ & $2.43 \pm 0.14 \mathrm{~b}-\mathrm{e}$ & $3.15 \pm 0.22 b-d$ \\
\hline & $4 \mathrm{~A} 313$ & $6.13 \pm 0.70 \mathrm{a}-\mathrm{d}$ & ND & 0.00 & $1.91 \pm 0.12 \mathrm{~h}-\mathrm{k}$ \\
\hline & $4 \mathrm{~A} 35$ & $5.00 \pm 0.58 \mathrm{a}-\mathrm{e}$ & ND & $1.58 \pm 0.11 \mathrm{~h}-\mathrm{k}$ & $2.79 \pm 0.18 b-f$ \\
\hline & $4 \mathrm{~B} 312$ & $3.42 \pm 0.48 \mathrm{~d}-\mathrm{f}$ & $3.35 \pm 0.53 b c$ & $2.43 \pm 0.14 \mathrm{~b}-\mathrm{e}$ & $3.33 \pm 0.00 b$ \\
\hline & $4 \mathrm{~B} 314$ & $7.26 \pm 0.90 \mathrm{a}$ & ND & $1.08 \pm 0.12 \mathrm{jk}$ & $1.83 \pm 0.04 \mathrm{i}-\mathrm{k}$ \\
\hline & 4B37 & $4.17 \pm 0.48 \mathrm{~b}-\mathrm{f}$ & ND & $1.72 \pm 0.06 \mathrm{f}-\mathrm{j}$ & $2.87 \pm 0.07 \mathrm{~b}-\mathrm{e}$ \\
\hline & 4B38 & $6.22 \pm 0.95 \mathrm{a}-\mathrm{c}$ & $2.5 \pm 0.28 \mathrm{~cd}$ & $1.67 \pm 0.00 \mathrm{~g}-\mathrm{k}$ & $2.40 \pm 0.05 \mathrm{~d}-\mathrm{k}$ \\
\hline & 4B39 & 0.00 & $7.93 \pm 0.86 \mathrm{a}$ & $1.89 \pm 0.00 \mathrm{e}-\mathrm{j}$ & $1.67 \pm 0.00 \mathrm{j}-\mathrm{k}$ \\
\hline & $5 \mathrm{~A} 32$ & $4.86 \pm 0.22 \mathrm{a}-\mathrm{e}$ & $2.06 \pm 0.48 \mathrm{~cd}$ & $2.16 \pm 0.08 \mathrm{e}-\mathrm{i}$ & $2.71 \pm 0.35 \mathrm{~b}-\mathrm{h}$ \\
\hline & $5 \mathrm{~A} 36$ & ND & 0.00 & $2.52 \pm 0.29 \mathrm{~b}-\mathrm{d}$ & $2.5 \pm 0.00 \mathrm{c}-\mathrm{i}$ \\
\hline & $5 \mathrm{~B} 31$ & $4.7 \pm 0.22 \mathrm{a}-\mathrm{e}$ & ND & $2.16 \pm 0.18 \mathrm{e}-\mathrm{i}$ & $3.38 \pm 0.09 b$ \\
\hline & $5 \mathrm{~B} 312$ & $6.16 \pm 0.71 \mathrm{a}-\mathrm{c}$ & 0.00 & ND & ND \\
\hline & $5 \mathrm{~B} 313$ & $5.40 \pm 0.00 \mathrm{a}-\mathrm{e}$ & 0.00 & $3.14 \pm 0.14 \mathrm{a}$ & $2.46 \pm 0.18 \mathrm{c}-\mathrm{j}$ \\
\hline & $5 \mathrm{~B} 323$ & $6.24 \pm 0.88 \mathrm{a}-\mathrm{c}$ & ND & $1.66 \pm 0.11 \mathrm{~g}-\mathrm{k}$ & $2.05 \pm 0.10 \mathrm{f}-\mathrm{k}$ \\
\hline & $5 \mathrm{~B} 328$ & $5.92 \pm 0.38 \mathrm{a}-\mathrm{d}$ & $3.03 \pm 0.25 \mathrm{~b}-\mathrm{d}$ & 0.00 & 0.00 \\
\hline & $5 \mathrm{~B} 329$ & ND & $8.21 \pm 0.74 \mathrm{a}$ & $2.00 \pm 0.08 \mathrm{~d}-\mathrm{j}$ & $1.61 \pm 0.04 \mathrm{kl}$ \\
\hline & $5 \mathrm{~B} 33$ & $4.44 \pm 0.38 \mathrm{~b}-\mathrm{f}$ & ND & $2.16 \pm 0.08 \mathrm{e}-\mathrm{i}$ & $2.71 \pm 0.20 \mathrm{~b}-\mathrm{h}$ \\
\hline & $5 \mathrm{~B} 336$ & ND & ND & $2.78 \pm 0.19 a-c$ & $2.33 \pm 0.16 \mathrm{e}-\mathrm{k}$ \\
\hline & $5 \mathrm{~B} 337$ & $3.77 \pm 0.37 \mathrm{c}-\mathrm{f}$ & ND & $2.50 \pm 0.00 \mathrm{~b}-\mathrm{e}$ & $2.42 \pm 0.07 \mathrm{~d}-\mathrm{k}$ \\
\hline & $5 \mathrm{~B} 340$ & $6.60 \pm 0.40 \mathrm{ab}$ & ND & 0.00 & $1.86 \pm 0.02 \mathrm{i}-\mathrm{k}$ \\
\hline
\end{tabular}


Table 2 continued

\begin{tabular}{|c|c|c|c|c|c|}
\hline \multirow[t]{2}{*}{ Order } & \multirow[t]{2}{*}{ Isolate } & \multicolumn{4}{|l|}{ Enzymatic activity } \\
\hline & & Amilase & Protease & Esterase & Lipase \\
\hline \multirow[t]{24}{*}{ Actinomicetales } & $1 \mathrm{~A} 358$ & $3.35 \pm 0.34 \mathrm{ab}$ & 0.00 & 0.00 & 0.00 \\
\hline & $1 \mathrm{~A} 373$ & 0.00 & 0.00 & 0.00 & $4.07 \pm 0.18 \mathrm{ab}$ \\
\hline & $1 \mathrm{~B} 18 \mathrm{~S}$ & $2.80 \pm 0.14 \mathrm{~b}-\mathrm{f}$ & 0.00 & 0.00 & $4.25 \pm 1.25 \mathrm{ab}$ \\
\hline & $1 \mathrm{~B} 5 \mathrm{~S}$ & $2.28 \pm 0.25 \mathrm{e}-\mathrm{h}$ & $2.43 \pm 0.20 \mathrm{ef}$ & $2.77 \pm \mathbf{0 . 1 5} b$ & $2.29 \pm 0.17 \mathrm{c}-\mathrm{e}$ \\
\hline & $1 \mathrm{~B} 7 \mathrm{~S}$ & $3.25 \pm 0.05 \mathrm{a}-\mathrm{c}$ & 0.00 & 0.00 & $4.00 \pm 0.00 \mathrm{ab}$ \\
\hline & $2 \mathrm{~A} 13 \mathrm{~S}$ & $3.26 \pm 0.08$ a-c & $3.71 \pm 0.29 \mathrm{a}-\mathrm{d}$ & 0.00 & $4.83 \pm 0.16 \mathrm{a}$ \\
\hline & $2 \mathrm{~A} 1 \mathrm{~S}$ & 0.00 & $3.08 \pm 0.14 \mathrm{a}-\mathrm{e}$ & 0.00 & 0.00 \\
\hline & $2 \mathrm{~A} 21 \mathrm{~S}$ & 0.00 & $3.71 \pm 0.29 \mathrm{a}-\mathrm{d}$ & 0.00 & $3.95 \pm 0.12 \mathrm{ab}$ \\
\hline & $2 \mathrm{~A} 23 \mathrm{~S}$ & $3.22 \pm 0.10 \mathrm{a}-\mathrm{d}$ & 0.00 & 0.00 & $1.94 \pm 0.22 \mathrm{c}-\mathrm{e}$ \\
\hline & $2 \mathrm{~A} 324$ & $3.05 \pm 0.05 \mathrm{a}-\mathrm{e}$ & 0.00 & 0.00 & $3.83 \pm 0.23 a b$ \\
\hline & $2 \mathrm{~A} 370$ & $3.20 \pm 0.14 \mathrm{a}-\mathrm{d}$ & 0.00 & 0.00 & $4.25 \pm 0.25 \mathrm{ab}$ \\
\hline & $3 \mathrm{~A} 1 \mathrm{~S}$ & 0.00 & $3.23 \pm 0.22 \mathrm{a}-\mathrm{e}$ & 0.00 & 0.00 \\
\hline & $3 \mathrm{~A} 35$ & $2.28 \pm 0.14 \mathrm{e}-\mathrm{h}$ & $3.00 \pm 0.09 \mathrm{~b}-\mathrm{e}$ & 0.00 & 0.00 \\
\hline & $3 \mathrm{~B} 2 \mathrm{~S}$ & $1.92 \pm 0.04 \mathrm{gh}$ & $4.02 \pm 0.18$ ab & 0.00 & 0.00 \\
\hline & 3B311 & $3.25 \pm 0.09 \mathrm{a}-\mathrm{c}$ & 0.00 & 0.00 & $3.08 \pm 0.08 \mathrm{~b}-\mathrm{d}$ \\
\hline & $3 \mathrm{~B} 320$ & 0.00 & 0.00 & 0.00 & $3.84 \pm 0.27 \mathrm{ab}$ \\
\hline & $4 \mathrm{~A} 11 \mathrm{~S}$ & 0.00 & $4.28 \pm 0.46 \mathrm{a}$ & $3.37 \pm 0.34$ a & 0.00 \\
\hline & $4 \mathrm{~A} 353$ & $2.79 \pm 0.18 \mathrm{~b}-\mathrm{f}$ & 0.00 & 0.00 & $4.22 \pm 0.25 \mathrm{ab}$ \\
\hline & $5 \mathrm{~A} 2 \mathrm{~S}$ & 0.00 & $3.71 \pm 0.41 \mathrm{a}-\mathrm{d}$ & $3.02 \pm 0.23 \mathrm{ab}$ & 0.00 \\
\hline & $5 \mathrm{~A} 4 \mathrm{~S}$ & $3.64 \pm 0.14 \mathrm{a}$ & 0.00 & 0.00 & 0.00 \\
\hline & $5 \mathrm{~A} 5 \mathrm{~S}$ & 0.00 & $3.79 \pm 0.12 \mathrm{a}-\mathrm{c}$ & 0.00 & 0.00 \\
\hline & $5 \mathrm{~B} 2 \mathrm{~S}$ & 0.00 & $3.28 \pm 0.27 \mathrm{a}-\mathrm{e}$ & 0.00 & 0.00 \\
\hline & $5 \mathrm{~B} 3 \mathrm{~S}$ & $2.44 \pm 0.23 \mathrm{~d}-\mathrm{g}$ & $4.07 \pm 0.34$ ab & 0.00 & 0.00 \\
\hline & $5 \mathrm{~B} 5 \mathrm{~S}$ & $3.11 \pm 0.35 \mathrm{a}-\mathrm{d}$ & 0.00 & 0.00 & $3.30 \pm 0.12 b c$ \\
\hline \multirow[t]{12}{*}{ Bacillales } & $1 \mathrm{~A} 33$ & $5.06 \pm 0.33$ a & $4.67 \pm 0.18 \mathrm{ab}$ & $4.67 \pm 0.18 \mathrm{ab}$ & $1.48 \pm 0.05 \mathrm{~d}$ \\
\hline & $1 \mathrm{~A} 337$ & $2.86 \pm 0.33 \mathrm{ab}$ & $5.53 \pm 0.36$ a & 0.00 & 0.00 \\
\hline & $1 \mathrm{~A} 339$ & $3.75 \pm 0.66 \mathrm{ab}$ & $3.74 \pm 0.06 \mathrm{bc}$ & $1.16 \pm 0.03 \mathrm{~d}$ & $3.86 \pm 0.18$ a \\
\hline & $1 \mathrm{~A} 364$ & 0.00 & 0.00 & 0.00 & $0.00 \pm 0.00 \mathrm{e}$ \\
\hline & $1 \mathrm{~B} 39$ & $4.83 \pm 0.73 a$ & $5.36 \pm 0.13$ a & $1.18 \pm 0.23 \mathrm{~cd}$ & $1.73 \pm 0.24 \mathrm{~cd}$ \\
\hline & $2 \mathrm{~A} 349$ & $3.62 \pm 0.53 \mathrm{ab}$ & $4.04 \pm 0.35 \mathrm{a}-\mathrm{c}$ & $1.50 \pm 0.17 \mathrm{~b}-\mathrm{d}$ & $1.47 \pm 0.14 \mathrm{~d}$ \\
\hline & $2 \mathrm{~B} 35$ & $3.20 \pm 0.33 \mathrm{ab}$ & $2.84 \pm 0.04 \mathrm{c}$ & $1.15 \pm 0.03 \mathrm{~d}$ & $1.97 \pm 0.03 \mathrm{~cd}$ \\
\hline & $3 \mathrm{~A} 312$ & $4.09 \pm 0.86 \mathrm{ab}$ & $5.25 \pm 0.36$ ab & $1.32 \pm 0.08 \mathrm{~cd}$ & $1.45 \pm 0.13 \mathrm{~d}$ \\
\hline & $4 \mathrm{~B} 2 \mathrm{~S}$ & $2.33 \pm 0.59 \mathrm{~b}$ & $5.11 \pm 0.33 \mathrm{ab}$ & $2.34 \pm \mathbf{0 . 0 8} a$ & $2.68 \pm 0.20 \mathrm{~b}$ \\
\hline & $5 \mathrm{~A} 1 \mathrm{~S}$ & 0.00 & 0.00 & 0.00 & 0.00 \\
\hline & $5 \mathrm{~A} 312$ & 0.00 & $4.63 \pm 0.67 \mathrm{ab}$ & $1.80 \pm 0.04 \mathrm{a}-\mathrm{c}$ & $1.33 \pm 0.01 \mathrm{~d}$ \\
\hline & $5 \mathrm{~B} 312$ & 0.00 & 0.00 & $1.97 \pm 0.04$ ab & $2.33 \pm 0.16 \mathrm{bc}$ \\
\hline
\end{tabular}

The isolates classified as the ten higher producers are shown in bold; $N D$ means not determined

industrial and biotechnological applications. The availability of nutrients and content of organic matter are variable in the mangrove. In marine sediments, Microbacterium spp. and Vibrio spp. have been found to be associated with marine animals where they contribute to the decomposition of biological molecules (Yu et al. 2004).

Isolates affiliated to Vibrionales appeared to be the predominant enzyme-producing group within the community when compared with other groups (Actinomycetales and Bacillales), mainly for the production of amylase and protease. The other enzymes could be produced in equal amounts by the three groups (Table 2). The order Vibrionales revealed to be metabolically versatile, with a high production of enzymes. Previous studies also indicated that Vibrionales was an enzyme-producing group, and an isolate of Vibrio fluvialis from mangrove sediments was used to produce an alkaline extracellular protease with high efficiency for use in industrial detergents (Venugopal and 
Saramma 2006). Although the Vibrionales were the major enzymatic producers, other isolated bacteria (Actinomycetales and Bacillales) also demonstrated the ability to produce targeted enzymes, albeit at lower levels.

An extremely high production of the enzymes by the isolates was not observed. Hence, the data present isolates with enzymatic production in the mangrove, where exceptional environmental conditions occur, like the higher salinity and the low availability of oxygen. Considering that, these enzymes could be further explored in their differential functionality, and for genetic breeding leading to higher production levels.

Acknowledgments This work was funded by financial support from the FAPESP/BIOTA (grant 04/13910-6 awarded to ISM, fellowship 06/57060-1 awarded to PTL and fellowship 07/56360-4 awarded to FDA) and CNPq (fellowship awarded to ACFD).

\section{References}

Almeida MA, Cunha MA, Dias JM (2007) Bacterial productivity distribution during a rainy year in an estuarine system. Microb Ecol 53:208-220. doi:10.1007/s00248-005-0082-6

Hankin L, Anagnostakis SL (1975) The use of solid media for detection of enzyme production by fungi. Mycologia 67:597607. doi: $10.2307 / 3758395$

Heuer H, Krsek M, Baker P, Smalla K, Wellington EMH (1997) Analysis of actinomycete communities by specific amplification of genes encoding 16S rRNA and gel-electrophoretic separation in denaturing gradients. Appl Environ Microbiol 63:3233-3241

Heyrman J, Mergaert J, Denys R, Swings J (1999) The use of fatty acid methyl ester analysis (FAME) for the identification of heterotrophic bacteria present on three mural paintings showing severe damage by microorganisms. FEMS Microbiol Lett 181:55-62. doi:10.1111/j.1574-6968.1999.tb08826.x

Holguin G, Bashan Y (1996) Nitrogen-fixation by Azospirillum brasilense $\mathrm{CD}$ is promoted when co-cultured with a mangrove rhizosphere bacterium Staphylococcus sp. Soil Biol Biochem 28:1651-1660. doi:10.1016/S0038-0717(96)00251-9

Holguin G, Zamorano PG, De-Bashan LE, Mendoza R, Amador E, Bashan Y (2006) Mangrove health in an arid environment encroached by urban development-a case study. Sci Total Environ 363:260-274. doi:10.1016/j.scitotenv.2005.05.026

Kathiresan K, Selvam MM (2006) Evaluation of beneficial bacteria from mangrove soil. Bot Mar 49:86-88. doi:10.1515/BOT. 2006.011
Lageiro MM, Moura MJ, Reis A, Ferreira MJC (2007) Microbial proteases application in leather industry. J Biotechnol 131:S239_ S240. doi:10.1016/j.jbiotec.2007.07.717

Schulze AD, Alabi AO, Sheldrake ART, Miller KM (2006) Bacterial diversity in a marine hatchery: balance between pathogenic and potentially probiotic bacterial strains. Aquaculture 256:50-73. doi:10.1016/j.aquaculture.2006.02.008

Sierra GA (1957) A simple method for the detection of lypolytic activity of microorganisms and some observations on the influence of the contact between cells and fatty substracts. Anton Lee 28:15-22. doi:10.1007/BF02545855

Sivaramakrishnan S, Gangadharan D, Nampoothiri KM, Soccol CR, Pandey A (2006) $\alpha$-Amylases from microbial sources-an overview on recent developments. Food Technol Biotechnol 44:173184

Smibert RM, Krieg NR (1994) Phenotypic characterization. In: Gerhardt P, Murray RGE, Wood WA, Krieg NR (eds) Methods for general and molecular bacteriology. American Society for Microbiology, Washington, pp 607-654

Sousa OV, Macrae A, Menezes FGR, Gomes NCM, Vieira RHSF, Mendonça-Hagler LCS (2006) The impact of shrimp farming effluent on bacterial communities in mangrove waters, Ceara, Brazil. Mar Pollut Bull 52:1725-1734. doi:10.1016/ j.marpolbul.2006.07.006

Stevens H, Brinkhoff T, Rink B, Vollmers J, Simon M (2007) Diversity and abundance of gram positive bacteria in a tidal flat ecosystem. Environ Microbiol 9:1810-1822

Thompson FL, Iida T, Swings J (2004) Biodiversity of Vibrios. Microbiol Mol Biol Rev 68:403-431. doi:10.1128/ MMBR.68.3.403-431.2004

Tiago I, Chung AP, Verissimo A (2004) Bacterial diversity in a nonsaline alkaline environment: heterotrophic aerobic populations. Appl Environ Microbiol 70:7378-7387. doi:10.1128/ AEM.70.12.7378-7387.2004

Venugopal M, Saramma AV (2006) Characterization of alkaline protease from Vibrio fluvialis strain VM10 isolated from a mangrove sediment sample and its application as a laundry detergent additive. Process Biochem 41:1239-1243. doi:10.1016/j.procbio.2005.12.025

Yu CF, Yu PHF, Chan PL, Yan Q, Wong PK (2004) Two novel species of tetrodotoxin-producing bacteria isolated from toxic marine puffer fishes. Toxicon 44:641-647. doi:10.1016/ j.toxicon.2004.07.021

Zhou HW, Guo CL, Wong YS, Tam NFY (2006) Genetic diversity of dioxygenase genes in polycyclic aromatic hydrocarbon-degrading bacteria isolated from mangrove sediments. FEMS Microbiol Lett 262:148-157 